\title{
Synthesis and biological evaluation of novel 4-0xo-5-cyano thiouracil derivatives as SecA inhibitors
}

https://doi.org/10.1515/hc-2020-0100

Received November 06, 2019; accepted March 11, 2020.

Abstract: The continuous emergence of drug-resistant strains of bacteria poses an urgent risk to human health and dictates the need for new antimicrobials. Along this line, we have been working on developing inhibitors of SecA, a key component of the bacterial Sec-dependent secretion machinery. Herein, we describe the synthesis and antimicrobial evaluation of 6-oxo-1,6-dihydropyrimidine-5-carbonitrile derivatives as potential SecA inhibitors.

Keywords: SecA inhibitor, small molecule, antimicrobial, target, drug-resistant

\section{Introduction}

Bacterial infection remains a serious threat to human health, largely because of the continuous emergence of drug-resistance strains of bacteria [1, 2]. To address this concern, both the World Health Organization (WHO) [3] and the US Center for Disease Control (CDC) [4] issued calls for urgent actions. Therefore, there has been much effort in search of new antimicrobials. Especially desirable are efforts toward identifying and inhibiting new targets and/ or new mechanisms of actions. Along this line, we have been working on developing SecA inhibitors as a way to achieve antimicrobial activities. SecA is a key component of the bacterial protein secretion (Sec) pathways. It is an
ATPase-driven "motor," which couples the hydrolysis of ATP to the stepwise translocation of preproteins across the bacterial cytoplasmic membrane [5-8]. Because SecA is a conserved and essential protein in all bacteria and is absent in humans, it is considered as a promising antibacterial drug target. Various SecA inhibitors (Figure 1) have been described in the literature [9]. Prior to our recent efforts, sodium azide was known as a SecA inhibitor and was reported to possess antibacterial activity with $\mathrm{IC}_{50}$ of $1 \mathrm{mM}$. The first natural product inhibitor of SecA, CJ-21058 [10] isolated from an unidentified fungus, showed antibacterial activity against Gram-positive bacteria by inhibiting the SecA translocation ATPase activity. Additional examples include pannomycin [11], thiazolo[4,5-d]pyrimidine derivatives [12], and others [13, 14]. During our studies on developing small molecule SecA inhibitors, we reported a structure-based virtual screening approach for the discovery of small-molecule inhibitors of the intrinsic ATPase activity of SecA [15]. Later on, we described optimization work [16] and inhibitors of different structural scaffolds [17, 18]. Among all the efforts, we reported 4-oxo-5-cyano thiouracil derivatives as SecA inhibitors [19]. Herein, we describe our efforts in optimizing a hit (Figure 1), a 4-oxo-5-cyano thiouracil, and evaluation of new analogs for antibacterial activities. The work is based on the general structural scaffold of the lead with the aim of searching for the surrounding chemical space [16]. Below, we describe the results and implications in guiding future work in this area.

\section{Results and discussion}

\section{Chemistry}

Fante Bamba, Departments of Chemistry, Georgia State University, Atlanta, Georgia 30303, USA; Laboratoire de Chimie Organique et des Substances Naturelles, Université Félix Houphouët-Boigny, 22 Bp 582 Abidjan 22, COTE D'IVOIRE

Jinshan Jin, Departments of Biology, Georgia State University, Atlanta, Georgia 30303, USA

*Corresponding authors: Phang C. Tai and Binghe Wang, Departments of Chemistry and Center for Diagnostics and Therapeutics, Georgia State University, Atlanta, Georgia 30303.

¿ Open Access. () 2020 Bamba et al., published by De Gruyter. Attribution alone 4.0 License.
Based on our earlier work [19], the compound in Figure 1 was the lead compound for further exploration of the chemical space that could inhibit SecA. In this respect, we focused our attention on the para position of the phenyl 


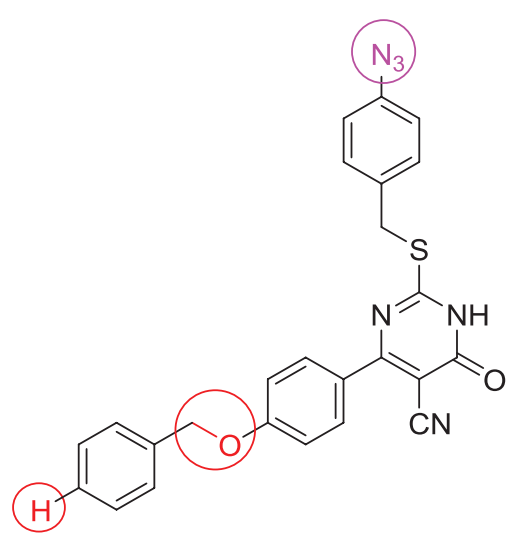

Figure 1 Lead compound for the synthesis of novel SecA inhibitors. group, para position of the benzylthio group and the linker between the biphenyl rings. Specifically, we designed compounds with various substituents such as F-, Me-, $\mathrm{MeO}$ - on the phenyl group at position 4; linkers such as $-\mathrm{CH}_{2} \mathrm{O}-$, - $\mathrm{O}-$, or - $\mathrm{HN}-\mathrm{CO}-$ between the two phenyl rings, and substituents such as $-\mathrm{N}_{3}$, -COOMe, or $-\mathrm{CF}_{3}$ at the 4-position of the benzylthioether moiety. Such variations led to compounds 3a-w.

The synthetic route of the compounds 3a-w is outlined in Scheme 1. Compounds 1a-e were prepared by $O$-benzylation of 4-hydroxybenzaldehyde using the appropriate aryl halides under basic conditions [20]. The corresponding 4-formylbenzoic acid was coupled with the

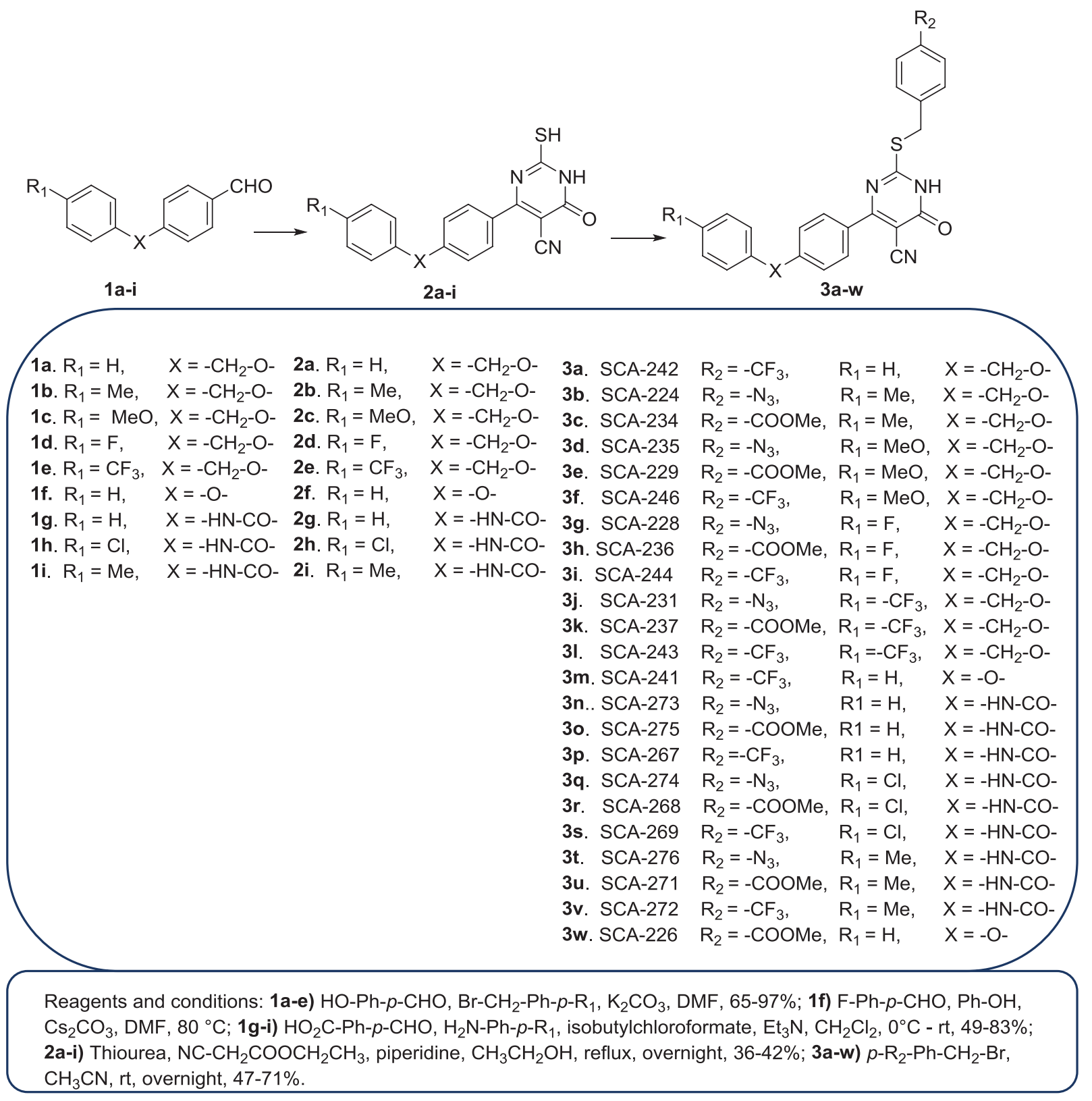

Scheme 1 Syntheses of compounds 3a-w. 
respective phenyl/aryl amines using isobutyl chloroformate in the presence of triethylamine to provide amide of type 1g-h by published procedure [21]. Following the methods for obtaining the core scaffold previously [16], compounds 2a-I were obtained by condensation of an appropriate aldehyde with ethyl cyanoacetate and thiourea in the presence of piperidine. This was followed by the $S$-benzylation in the presence of potassium carbonate in acetonitrile to obtain compounds 3a-w.

\section{Biological evaluation}

The activities of the synthesized compounds were evaluated in a MIC assay using established procedures using a mutant of Escherichia. coli with a compromised outer membrane [19]. It was found that most of the compounds were not active at concentrations as high as $250 \mu \mathrm{M}$. Three compounds showed MIC at about $20 \mu \mathrm{M}(\mathbf{3 i}, \mathbf{l}, \mathbf{w})$, and one at about $8 \mu \mathrm{M}(3 \mathrm{~m})$, which is comparable to some of our best compounds in this class [22]. In addition, select compounds were also tested against Gram-positive bacteria Bacillus anthracis (Sterne strain). With the limited data set, it seems that the potency against $B$. anthracis was comparable with or higher than that of $E$. coli. For example, the MIC of $\mathbf{3 w}$ was $25 \mu \mathrm{M}$ against $E$. coli and $12.5 \mu \mathrm{M}$ against $B$. anthracis. By examining the details, it seems that a $\mathrm{CF}_{3}$ group at the $\mathrm{R}_{2}$ position is beneficial, especially if the central linker is $-\mathrm{CH}_{2}-\mathrm{O}$-. With a -O- linker, it seems that an ester group also offers a good outcome. Overall, the results are very useful information for those who might be interested in SecA inhibitor for both improved potencies and for avoiding chemical space that would not be productive.

\section{Conclusion}

We have described the design, syntheses and biological evaluation of a novel structural class of small-molecule SecA inhibitors with $\mu \mathrm{M}$ inhibition. $\mathrm{A} \mathrm{CF}_{3}$ group at the $\mathrm{R}_{2}$ position and a diaryl ether linker seem to be beneficial for improved potency of the lead compound.

\section{Experimental}

All chemical reagents and solvents used were reagent grade or purified using standard methods. TLC analyses were conducted on silica gel plates (Sorbent Silica G
UV254). Column chromatography was carried out on flash silica gel (Sorbent 230-400 mesh). NMR spectra were recorded at ${ }^{1} \mathrm{H}(400 \mathrm{MHz})$ and ${ }^{13} \mathrm{C}(100 \mathrm{MHz})$ on a Bruker instrument. Coupling constants $(J)$ and chemical shifts ( $\delta)$ are given in hertz and ppm respectively, using TMS $\left({ }^{1} \mathrm{H}\right.$ NMR) and solvents ( ${ }^{13} \mathrm{C}$ NMR) as internal standards.

General procedure for the synthesis of (2a-i): Our previously published [24] procedure was followed. Briefly, to a solution of ethanol $(25 \mathrm{~mL})$ and appropriate aldehyde (RCHO, $5 \mathrm{mmol}$ ) was added ethyl cyanoacetate $(0.5 \mathrm{~mL}, 5$ $\mathrm{mmol})$, thiourea (0.38 g, $5 \mathrm{mmol}$ ) and piperidine $(1.0 \mathrm{~mL}$, $10 \mathrm{mmol})$. The mixture was heated under reflux overnight and then cooled to room temperature. The precipitate was dissolved in $0.5 \mathrm{M} \mathrm{NaOH}(20 \mathrm{~mL})$ and washed with ethyl acetate $(15 \mathrm{ml} \times 3)$. Then the aqueous solution was acidified to $\mathrm{pH} \sim 2$ by slow addition of $1 \mathrm{M} \mathrm{HCl}$. This caused the product to precipitate, which was then filtered using vacuum filtration.

General procedure for the synthesis of (3a-w): To a solution of 4-(4-(benzyloxy)phenyl)-2-mercapto6-oxo-1,6-dihydropyrimidine-5-carbonitrile derivatives $(1.36 \mathrm{mmol})$ in $\mathrm{CH}_{3} \mathrm{CN}(10 \mathrm{ml}), \mathrm{K}_{2} \mathrm{CO}_{3}(6.79 \mathrm{mmol})$ was added and the resulting mixture was stirred for 10-15 min. To this was added the appropriate (bromomethyl)phenyl derivatives $(1.22 \mathrm{mmol})$ and the reaction was stirred at room temperature for 16-18h. Upon disappearance of the starting material, the solvent was removed in vacuo. The dried residue was washed by $\mathrm{H}_{2} \mathrm{O}(\mathrm{pH}=9-10,20 \mathrm{~mL} \times 2)$ and brine $(15 \mathrm{ml} \times 2)$ followed by product extraction in ethyl acetate $(20 \mathrm{ml})$. The solvent was evaporated in vacuo to obtain crude product, which was purified using silica gel column chromatography.

\section{2-mercapto-4-(4-((4-methylbenzyl)oxy)phenyl)-6-oxo- 1,6-dihydropyrimidine-5-carbonitrile (2b)}

Yield 37\%; white solid; ${ }^{1} \mathrm{H}$ NMR (DMSO- $d_{6}$ ): $\delta 13.16$ (bs, $1 \mathrm{H}$ ), $12.86(\mathrm{~s}, 1 \mathrm{H}), 7.66$ (d, $J=8.8 \mathrm{~Hz}, 2 \mathrm{H}), 7.37$ (d, $J=8.1 \mathrm{~Hz}, 2 \mathrm{H})$, $7.22(\mathrm{~d}, J=7.6 \mathrm{~Hz}, 2 \mathrm{H}), 7.16(\mathrm{~d}, J=8.4 \mathrm{~Hz}, 2 \mathrm{H}), 5.16(\mathrm{~s}, 2 \mathrm{H}) ;{ }^{13} \mathrm{C}$ NMR (DMSO- $d_{6}$ ): $\delta$ 177.5, 161.7, 159.7, 137.8, 133.9, 131.2, 129.5, 128.4, 122.8, 116.1, 115.1, 89.5, 69.9, 21.3. HRMS (ESI) (m/z): Calcd. for $\mathrm{C}_{19} \mathrm{H}_{14} \mathrm{~N}_{3} \mathrm{O}_{2} \mathrm{~S},[\mathrm{M}-\mathrm{H}]^{+}$: 348.0803 ; found: 348.0803 .

\section{2-mercapto-4-(4-((4-methoxybenzyl)oxy)phenyl)-6-oxo- 1,6-dihydropyrimidine-5-carbonitrile (2c)}

Yield 42\%; white solid; ${ }^{1} \mathrm{H}$ NMR (DMSO- $d_{6}$ ): $\delta 13.15$ (bs, $1 \mathrm{H}$ ), $12.85(\mathrm{~s}, 1 \mathrm{H}), 7.66$ (d, $J=8.8 \mathrm{~Hz}, 2 \mathrm{H}), 7.41(\mathrm{~d}, J=8.4 \mathrm{~Hz}, 2 \mathrm{H})$, $7.16(\mathrm{~d}, J=8.4 \mathrm{~Hz}, 2 \mathrm{H}), 6.96(\mathrm{~d}, J=8.4 \mathrm{~Hz}, 2 \mathrm{H}), 5.12(\mathrm{~s}, 2 \mathrm{H})$, 
$3.76(\mathrm{~s}, 3 \mathrm{H}) ;{ }^{13} \mathrm{C}$ NMR (DMSO-d $)$ : $\delta 177.4,161.7,161.6,159.6$, 159.6, 131.2, 130.1, 128.7, 22.7, 116.1, 115.0, 114.3, 89.4, 69.7, 55.5. HRMS (ESI) (m/z): Calcd. for $\mathrm{C}_{19} \mathrm{H}_{14} \mathrm{~N}_{3} \mathrm{O}_{3} \mathrm{~S}$, [M-H]+: 364.0750; found: 364.0753 .

\section{4-(4-((4-fluorobenzyl)oxy)phenyl)-2-mercapto-6-oxo-} 1,6-dihydropyrimidine-5-carbonitrile (2d)

Yield 40\%; white solid; ${ }^{1} \mathrm{H}$ NMR (DMSO- $\left.d_{6}\right): \delta 13.17$ (bs, $1 \mathrm{H}$ ), 12.84 (s, 1H), 7.68 (d, $J=8.0 \mathrm{~Hz}, 2 \mathrm{H}), 7.54$ (m, 2H), 7.24 (t, $J=$ $8.8 \mathrm{~Hz}, 2 \mathrm{H}), 7.18$ (d, $J=8.8 \mathrm{~Hz}, 2 \mathrm{H}), 5.19(\mathrm{~s}, 2 \mathrm{H}) ;{ }^{13} \mathrm{C}$ NMR $\left(\right.$ DMSO- $\left.d_{6}\right): \delta 177.4,163.6,161.4,159.5,133.2,131.1,130.6$, 115.8, 115.6, 114.9, 89.1, 69.2. HRMS (ESI) (m/z): Calcd. for $\mathrm{C}_{18} \mathrm{H}_{12} \mathrm{FN}_{3} \mathrm{O}_{2} \mathrm{~S},[\mathrm{M}-\mathrm{H}]^{+}:$352.0551; found: 352.0556 .

\section{2-mercapto-6-0xo-4-(4-((4-(trifluoromethyl)benzyl)oxy) phenyl)-1,6-dihydropyrimidine-5-carbonitrile (2e)}

Yield 36\%; white solid; ${ }^{1} \mathrm{H}$ NMR (DMSO- $d_{6}$ ): $\delta 13.18$ (bs, 1H), 12.73 (s, 1H), $7.79(\mathrm{~d}, J=8.0 \mathrm{~Hz}, 4 \mathrm{H}), 7.70(\mathrm{~m}, 4 \mathrm{H})$, 7.19 (d, $J=8.8 \mathrm{~Hz}, 2 \mathrm{H}), 5.34(\mathrm{~s}, 2 \mathrm{H}) ;{ }^{13} \mathrm{C}$ NMR (DMSO- $\left.d_{6}\right): \delta$ 177.8, 161.9, 161.2, 159.9, 141.9, 131.2, 129,1, 128.7, 128.6, 126.0, 125.8, 125.8, 123.6, 123.3, 116.3, 115.0, 89.2, 69.0. HRMS (ESI) (m/z): Calcd. for $\mathrm{C}_{19} \mathrm{H}_{11} \mathrm{~F}_{3} \mathrm{~N}_{3} \mathrm{O}_{2} \mathrm{~S},[\mathrm{M}-\mathrm{H}]^{+}$: 402.0519; found: 402.0522.

\section{4-(5-cyano-2-mercapto-6-oxo-1,6-dihydropyrimidin- 4-yl)-N-phenylbenzamide (2g)}

Yield 42\%; white solid; ${ }^{1} \mathrm{H}$ NMR (DMSO- $d_{6}$ ): $\delta 12.32(\mathrm{~s}, 1 \mathrm{H})$, 10.41 (s, $1 \mathrm{H}), 8.07$ (d, $J=8.4 \mathrm{~Hz}, 2 \mathrm{H}), 7.85$ (d, $J=8.0 \mathrm{~Hz}, 2 \mathrm{H})$, $7.80(\mathrm{~d}, J=8.0 \mathrm{~Hz}, 2 \mathrm{H}), 7.36(\mathrm{~d}, J=8.0 \mathrm{~Hz}, 2 \mathrm{H}), 7.10$ (t, $J=$ $7.2 \mathrm{~Hz}, 2 \mathrm{H}) ;{ }^{13} \mathrm{C}$ NMR (DMSO- $\left.d_{6}\right): \delta 180.1,167.6,165.3,163.9$, $160.9,141.1,139.4,137.5,136.6,129.5,129.1,129.0,128.2$, 128.0, 124.3, 120.9, 120.8, 118.6, 117.0, 88.8. HRMS (ESI) (m/z): Calcd. for $\mathrm{C}_{18} \mathrm{H}_{11} \mathrm{~N}_{4} \mathrm{O} 2 \mathrm{~S},[\mathrm{M}-\mathrm{H}]^{+}:$347.0597; found: 347.0599.

$\mathrm{N}$-(4-chlorophenyl)-4-(5-cyano-2-mercapto-6-oxo-1,6-dihydropyrimidin-4-yl)benzamide ( $2 \mathrm{~h})$

Yield 38\%; white solid; ${ }^{1} \mathrm{H}$ NMR (DMSO- $d_{6}$ ): $\delta 11.76(\mathrm{~s}, 1 \mathrm{H})$, 10.52 (s, 1H), 8.03 (d, $J=8.0 \mathrm{~Hz}, 2 \mathrm{H}), 7.86$ (m, 4H), 7.42 (d, $J=8.8 \mathrm{~Hz}, 2 \mathrm{H}$ ); ${ }^{13} \mathrm{C}$ NMR (DMSO- $\left.d_{6}\right): \delta 183.1,166.6,165.5$, 162.7, 140.3, 138.5, 136.3, 128.9, 128.7, 127.9, 122.4, 118.8, 86.2. HRMS (ESI) (m/z): Calcd. for $\mathrm{C}_{18} \mathrm{H}_{10} \mathrm{ClN}_{4} \mathrm{O}_{2} \mathrm{~S},[\mathrm{M}-\mathrm{H}]^{+}$: 381.0208; found: 381.0209 . 4-(5-cyano-2-mercapto-6-oxo-1,6-dihydropyrimidin4-yl)-N-(p-tolyl)benzamide (2i)

Yield 34\%; white solid; ${ }^{1} \mathrm{H}$ NMR (DMSO- $\left.d_{6}\right): \delta 11.68(\mathrm{~s}, 1 \mathrm{H}$ ), 10.29 (s, 1H), 8.02 (d, $J=8.4 \mathrm{~Hz}, 2 \mathrm{H}), 7.86$ (d, $J=8.4 \mathrm{~Hz}$, 2H), 7.68 (d, $J=8.4 \mathrm{~Hz}, 2 \mathrm{H}), 7.17$ (d, $J=8.4 \mathrm{~Hz}, 2 \mathrm{H}) ;{ }^{13} \mathrm{C}$ NMR (DMSO- $\left.d_{6}\right): \delta 183.6,167.0,165.2,162.7,140.8,137.0,136.6$, 133.2, 129.4, 128.6, 127.7, 120.9, 119.1, 86.0, 20.9. HRMS (ESI) (m/z): Calcd. for $\mathrm{C}_{19} \mathrm{H}_{13} \mathrm{~N}_{4} \mathrm{O}_{2} \mathrm{~S},[\mathrm{M}-\mathrm{H}]^{+}$: 361.0754; found: 361.0763 .

\section{4-(4-(benzyloxy)phenyl)-6-0xo-2-((4-(trifluoromethyl) benzyl)thio)-1,6-dihydropyrimidine-5-carbonitrile (3a)}

Yield 54\%; white solid; ${ }^{1} \mathrm{H}$ NMR (DMSO- $d_{6}$ ): $\delta 7.93$ (d, $J=8.4$ $\mathrm{Hz}, 2 \mathrm{H}), 7.66$ (qd, $J=8.0 \mathrm{~Hz}, 4 \mathrm{H}), 7.48$ (d, $J=7.2 \mathrm{~Hz}, 2 \mathrm{H}), 7.41$ (t, $J=7.2 \mathrm{~Hz}, 1 \mathrm{H}), 7.37(\mathrm{~m}, 1 \mathrm{H}), 7.18(\mathrm{~d}, J=8.4 \mathrm{~Hz}, 2 \mathrm{H}), 5.22$ (s, $2 \mathrm{H}), 4.61$ (s, $2 \mathrm{H}) ;{ }^{13} \mathrm{C}$ NMR (DMSO- $d_{6}$ ): $\delta$ 166.7, 165.5, 161.6, 142.5, 136.9, 131.2, 130.1, 128.9, 128.4, 128.3, 127.9, 126.0, 125.8, 125.7, 123.3, 116.9, 115.2, 91.9, 69.9, 33.8. HRMS (ESI) (m/z): Calcd. for $\mathrm{C}_{26} \mathrm{H}_{17} \mathrm{~F}_{3} \mathrm{~N}_{3} \mathrm{O}_{2} \mathrm{~S},[\mathrm{M}-\mathrm{H}]^{+}$: 492.0994; found: 492.0998.

2-((4-azidobenzyl)thio)-4-(4-((4-methylbenzyl)oxy) phenyl)-6-oxo-1,6-dihydropyrimidine-5-carbonitrile (3b)

Yield 61\%; white solid; ${ }^{1} \mathrm{H}$ NMR (DMSO- $\left.d_{6}\right): \delta 8.00(\mathrm{~d}, J=$ $8.4 \mathrm{~Hz}, 2 \mathrm{H}), 7.46$ (d, $J=8.0 \mathrm{~Hz}, 2 \mathrm{H}), 7.37$ (d, $J=8.0 \mathrm{~Hz}$, 2H), 7.20 (t, $J=8.8 \mathrm{~Hz}, 4 \mathrm{H}), 7.07$ (d, $J=8.4 \mathrm{~Hz}, 2 \mathrm{H}), 5.17$ (s, $2 \mathrm{H}), 4.54(\mathrm{~s}, 2 \mathrm{H}), 2.31(\mathrm{~s}, 3 \mathrm{H}) ;{ }^{13} \mathrm{C}$ NMR (DMSO- $\left.d_{6}\right): \delta 166.8$, $165.4,161.8,139.0,137.7,133.9,133.9,131.2,131.1,129.5$, 128.3, 127.7, 119.6, 116.7, 115.3, 92.0, 69.9, 34.0, 21.2. HRMS (ESI) (m/z): Calcd. for $\mathrm{C}_{26} \mathrm{H}_{19} \mathrm{~N}_{6} \mathrm{O}_{2} \mathrm{~S},[\mathrm{M}-\mathrm{H}]^{+}$: 479.1290; found: 479.1300 .

methyl4-(((5-cyano-4-(4-((4-methylbenzyl)oxy)phenyl)6-oxo-1,6-dihydropyrimidin-2-yl)thio)methyl)benzoate (3c)

Yield 55\%; white solid; ${ }^{1} \mathrm{H}$ NMR (DMSO- $\left.d_{6}\right): \delta 7.94$ (d, $J=$ $8.0 \mathrm{~Hz}, 2 \mathrm{H}), 7.90$ (d, $J=8.0 \mathrm{~Hz}, 2 \mathrm{H}), 7.57$ (d, $J=7.6 \mathrm{~Hz}, 2 \mathrm{H})$, 7.36 (d, $J=7.6 \mathrm{~Hz}, 2 \mathrm{H}), 7.21$ (d, $J=7.6 \mathrm{~Hz}, 2 \mathrm{H}), 7.17$ (d, $J=8.4$ $\mathrm{Hz}, 2 \mathrm{H}), 5.17$ (s, 2H), 4.60 (s, 2H), 3.83 (s, 3H), 2.31 (s, 3H); ${ }^{13} \mathrm{C}$ NMR (DMSO- $\left.d_{6}\right): \delta 166.8,166.4,165.5,161.7,137.7,133.9$, 131.2, 129.8, 129.7, 129.5, 129.0, 128.4, 127.8, 115.2, 91.8, 69.9, 52.5, 34.1, 21.2. HRMS (ESI) (m/z): Calcd. for $\mathrm{C}_{28} \mathrm{H}_{22} \mathrm{~N}_{3} \mathrm{O}_{4} \mathrm{~S}$, $[\mathrm{M}-\mathrm{H}]^{+}$: 496.1331; found: 496.1337. 
2-((4-azidobenzyl)thio)-4-(4-((4-methoxybenzyl)oxy) phenyl)-6-oxo-1,6-dihydropyrimidine-5-carbonitrile (3d)

Yield 50\%; white solid; ${ }^{1} \mathrm{H}$ NMR (DMSO- $\left.d_{6}\right): \delta 8.00(\mathrm{~d}, J=$ $8.4 \mathrm{~Hz}, 2 \mathrm{H}), 7.46(\mathrm{~d}, J=8.0 \mathrm{~Hz}, 2 \mathrm{H}), 7.41(\mathrm{~d}, J=8.4 \mathrm{~Hz}, 2 \mathrm{H})$, $7.19(\mathrm{~d}, J=8.4 \mathrm{~Hz}, 2 \mathrm{H}), 7.07(\mathrm{~d}, J=8.4 \mathrm{~Hz}, 2 \mathrm{H}), 6.96(\mathrm{~d}, J=$ $8.4 \mathrm{~Hz}, 2 \mathrm{H}), 5.14$ (s, 2H), 4.54 (s, 2H), 3.76 (s, 3H); ${ }^{13} \mathrm{C}$ NMR (DMSO-d $d_{6}$ ): $\delta$ 168.1, 166.6, 165.3, 161.9, 161.7, 159.6, 139.0, 133.9, 131.2, 131.1, 130.1, 128.8, 127.7, 119.7, 116.7, 115.3, 115.0, 114.3, 92.0, 69.8, 55.5, 34.0. HRMS (ESI) (m/z): Calcd. for $\mathrm{C}_{26} \mathrm{H}_{19} \mathrm{~N}_{6} \mathrm{O}_{3} \mathrm{~S},[\mathrm{M}-\mathrm{H}]^{+}$: 495.1239; found: 495.1249.

Methyl4-(((5-cyano-4-(4-((4-methoxybenzyl)oxy)phenyl)6-oxo-1,6-dihydropyrimidin-2-yl)thio)methyl)benzoate (3e)

Yield 49\%; white solid; ${ }^{1} \mathrm{H}$ NMR (DMSO- $\left.d_{6}\right): \delta 7.95$ (d, $J=$ $8.8 \mathrm{~Hz}, 2 \mathrm{H}), 7.90$ (d, $J=8.0 \mathrm{~Hz}, 4 \mathrm{H}), 7.57$ (d, $J=8.0 \mathrm{~Hz}, 2 \mathrm{H})$, $7.41(\mathrm{~d}, J=8.4 \mathrm{~Hz}, 2 \mathrm{H}), 7.17(\mathrm{~d}, J=8.4 \mathrm{~Hz}, 2 \mathrm{H}), 6.96(\mathrm{~d}, J=$ $8.4 \mathrm{~Hz}, 2 \mathrm{H}$ ), 5.13 (s, 2H), 4.60 (s, 2H), 3.83 (s, 3H), 3.76 (s, $3 \mathrm{H}) ;{ }^{13} \mathrm{C}$ NMR (DMSO- $d_{6}$ ): $\delta$ 166.6, 166.3, 165.2, 161.8, 159.6, 142.9, 131.2, 130.1, 128.8, 129.7, 129.1, 128.8, 127.6, 115.1, 114.3, 92.0, 69.8, 55.5, 52.6, 34.1. HRMS (ESI) (m/z): Calcd. for $\mathrm{C}_{28} \mathrm{H}_{22} \mathrm{~N}_{3} \mathrm{O}_{5} \mathrm{~S},[\mathrm{M}-\mathrm{H}]^{+}$: 512.1280; found: 522.1299.

4-(4-((4-methoxybenzyl)oxy)phenyl)-6-oxo-2-((4(trifluoromethyl)benzyl)thio)-1,6-dihydropyrimidine5-carbonitrile (3f)

Yield 54\%; white solid; ${ }^{1} \mathrm{H}$ NMR (DMSO- $\left.d_{6}\right): \delta 7.92(\mathrm{~d}, J=$ $8.4 \mathrm{~Hz}, 2 \mathrm{H}), 7.66$ (qd, $J=8.0 \mathrm{~Hz}, 4 \mathrm{H}), 7.41(\mathrm{~d}, J=8.4 \mathrm{~Hz}, 2 \mathrm{H})$, $7.16(\mathrm{~d}, J=8.8 \mathrm{~Hz}, 2 \mathrm{H}), 6.96(\mathrm{~d}, J=8.8 \mathrm{~Hz}, 2 \mathrm{H}), 5.12(\mathrm{~s}, 2 \mathrm{H})$, 4.60 (s, 2H), 3.73 (s, 3H); ${ }^{13} \mathrm{C}$ NMR (DMSO- $d_{6}$ ): $\delta 166.6,161.7$, 159.6, 142.6, 131.3, 130.1, 128.8, 128.5, 128.2, 127.9, 126.0, 125.8, 125.7, 117.1, 115.2, 114.3, 91.7, 69.7, 55.5, 30.9. HRMS (ESI) (m/z): Calcd. for $\mathrm{C}_{27} \mathrm{H}_{19} \mathrm{~F}_{3} \mathrm{~N}_{3} \mathrm{O}_{3},[\mathrm{M}-\mathrm{H}]^{+}$: 522.1099; found: 522.1090 .

2-((4-azidobenzyl)thio)-4-(4-((4-fluorobenzyl)oxy) phenyl)-6-oxo-1,6-dihydropyrimidine-5-carbonitrile (3g)

Yield 47\%; white solid; ${ }^{1} \mathrm{H}$ NMR (DMSO- $d_{6}$ ): $\delta 8.00$ (d, $J=$ $8.0 \mathrm{~Hz}, 2 \mathrm{H}), 7.54$ (t, $J=7.2 \mathrm{~Hz}, 4 \mathrm{H}), 7.46(\mathrm{~d}, J=7.6 \mathrm{~Hz}, 2 \mathrm{H})$, 7.25 (d, $J=8.4 \mathrm{~Hz}, 2 \mathrm{H}), 7.20$ (d, $J=8.8 \mathrm{~Hz}, 2 \mathrm{H}), 7.07$ (d, $J=$ $7.6 \mathrm{~Hz}, 2 \mathrm{H}$ ), 5.21 (s, 2H), 4.54 (s, 2H); ${ }^{13} \mathrm{C}$ NMR (DMSO- $d_{6}$ ): $\delta$ 166.6, 165.5, 163.5, 161.8, 161.6, 161.1, 139.1, 133.9, 133.2, 133.1, 131.3, 131.1, 130.6, 130.5, 127.9, 119.6, 116.7, 115.9, 115.6, 115.3, 115.1, 92.0, 69.3, 34.0. HRMS (ESI) (m/z): Calcd. for $\mathrm{C}_{25} \mathrm{H}_{16} \mathrm{FN}_{6} \mathrm{O}_{2} \mathrm{~S},[\mathrm{M}-\mathrm{H}]^{+}:$483.1039; found: 483.1031 .
Methyl 4-(((5-cyano-4-(4-((4-fluorobenzyl)oxy))phenyl)6-oxo-1,6-dihydropyrimidin-2-yl)thio)methyl)benzoate (3h)

Yield 63\%; white solid; ${ }^{1} \mathrm{H}$ NMR (DMSO- $d_{6}$ ): $\delta 7.94$ (d, $J=$ $8.8 \mathrm{~Hz}, 2 \mathrm{H}), 7.89$ (d, $J=8.0 \mathrm{~Hz}, 4 \mathrm{H}), 7.52-7.58$ (m, 4H), 7.25 $(\mathrm{t}, J=8.8 \mathrm{~Hz}, 2 \mathrm{H}), 7.17(\mathrm{~d}, J=8.8 \mathrm{~Hz}, 2 \mathrm{H}), 5.20$ (s, 2H), 4.58 (s, 2H), $3.84(\mathrm{~s}, 3 \mathrm{H}) ;{ }^{13} \mathrm{C}$ NMR (DMSO-d $d_{6}$ ): $\delta 166.6,166.4$, 163.5, 161.4, 143.4, 133.2, 131.1, 130.6, 130.5, 129.7, 129.7, 129.0, 128.2, 117.3, 115.8, 115.6, 115.1, 90.6, 69.2, 52.5, 34.1. HRMS (ESI) (m/z): Calcd. for $\mathrm{C}_{27} \mathrm{H}_{19} \mathrm{FN}_{3} \mathrm{O}_{4} \mathrm{~S},[\mathrm{M}-\mathrm{H}]^{+}:$500.1080; found:500.1093.

4-(4-((4-fluorobenzyl)oxy)phenyl)-6-oxo-2-((4(trifluoromethyl)benzyl)thio)-1,6-dihydropyrimidine5-carbonitrile (3i)

Yield 66\%; white solid; ${ }^{1} \mathrm{H}$ NMR (DMSO- $\left.d_{6}\right): \delta 7.92(\mathrm{~d}, J=$ $8.0 \mathrm{~Hz}, 2 \mathrm{H}), 7.94$ (qd, $J=8.0 \mathrm{~Hz}, 4 \mathrm{H}), 7.53(\mathrm{t}, J=6.4 \mathrm{~Hz}, 2 \mathrm{H})$, 7.23 (t, $J=8.4 \mathrm{~Hz}, 2 \mathrm{H}$ ), 7.17 (d, $J=8.4 \mathrm{~Hz}, 2 \mathrm{H}), 5.19$ (s, 2H), 4.58 (s, 2H); ${ }^{13}$ C NMR (DMSO- $d_{6}$ ): $\delta 166.7,166.2,163.5,161.4$, 161.1, 142.8, 133.2, 132.5, 131.1, 130.6, 130.5, 130.1, 128.2, 125.7, 117.3, 115.8, 115.6, 115.1, 90.6, 69.2, 33.8. HRMS (ESI) (m/z): Calcd. for $\mathrm{C}_{26} \mathrm{H}_{16} \mathrm{~F}_{4} \mathrm{~N}_{3} \mathrm{O}_{2} \mathrm{~S},[\mathrm{M}-\mathrm{H}]^{+}:$510.0899; found: 510.0896 .

2-((4-azidobenzyl)thio)-6-0xo-4-(4-((4-(trifluoromethyl) benzyl)oxy)phenyl)-1,6-dihydropyrimidine-5-carbonitrile (3i)

Yield 71\%; white solid; ${ }^{1} \mathrm{H}$ NMR (DMSO- $\left.d_{6}\right): \delta 8.01$ (d, $J=$ $8.8 \mathrm{~Hz}, 2 \mathrm{H}), 7.79$ (d, $J=8.0 \mathrm{~Hz}, 2 \mathrm{H}), 7.70(\mathrm{~d}, J=8.0 \mathrm{~Hz}, 2 \mathrm{H})$, $7.45(\mathrm{~d}, J=8.0 \mathrm{~Hz}, 2 \mathrm{H}), 7.22(\mathrm{~d}, J=8.4 \mathrm{~Hz}, 2 \mathrm{H}), 7.06(\mathrm{~d}, J=$ $8.4 \mathrm{~Hz}, 2 \mathrm{H}$ ), 5.36 (s, 2H), 4.53 (s, 2H); ${ }^{13} \mathrm{C}$ NMR (DMSO- $d_{6}$ ): $\delta$ 161.4, 141.8, 139.0, 133.9, 131.3, 131.1, 129.1, 128.6, 128.1, 125.8, 119.6, 116.7, 115.3, 115.1, 92.1, 69.0, 34.0. HRMS (ESI) (m/z): Calcd. for $\mathrm{C}_{26} \mathrm{H}_{16} \mathrm{~F}_{3} \mathrm{~N}_{6} \mathrm{O}_{2} \mathrm{~S},[\mathrm{M}-\mathrm{H}]^{+}$: 533.1008; found: 533.1001.

Methyl 4-(((5-cyano-6-0xo-4-(4-((4-(trifluoromethyl) benzyl)oxy)phenyl)-1,6-dihydropyrimidin-2-yl)thio) methyl)benzoate (3k)

Yield 52\%; white solid; ${ }^{1} \mathrm{H}$ NMR (DMSO- $\left.d_{6}\right): \delta 7.96$ (d, $J=$ $8.0 \mathrm{~Hz}, 2 \mathrm{H}), 7.89(\mathrm{~d}, J=8.0 \mathrm{~Hz}, 2 \mathrm{H}), 7.79(\mathrm{~d}, J=7.6 \mathrm{~Hz}$, $2 \mathrm{H}), 7.70(\mathrm{~d}, J=7.6 \mathrm{~Hz}, 2 \mathrm{H}), 7.56(\mathrm{~d}, J=8.0 \mathrm{~Hz}, 2 \mathrm{H}), 7.20$ (d, $J=8.0 \mathrm{~Hz}, 2 \mathrm{H}), 5.76$ (s, 2H), $4.60(\mathrm{~s}, 2 \mathrm{H}), 3.83$ (s, 3H); ${ }^{13}$ C NMR (DMSO- $\left.d_{6}\right): \delta 166.4,161.4,142.9,141.9,131.3,129.7$, 
129.7, 129.1, 128.6, 125.8, 125.8, 115.3, 92.1, 69.0, 55.3, 52.5, 34.1. HRMS (ESI) (m/z): Calcd. for $\mathrm{C}_{28} \mathrm{H}_{19} \mathrm{~F}_{3} \mathrm{~N}_{3} \mathrm{O}_{4} \mathrm{~S}$, [M-H] 550.1048; found: 550.1039.

6-oxo-4-(4-((4-(trifluoromethyl)benzyl)oxy)phenyl)-2((4-(trifluoromethyl)benzyl)thio)-1,6-dihydropyrimidine5-carbonitrile (3l)

Yield 68\%; white solid; ${ }^{1} \mathrm{H}$ NMR (DMSO- $d_{6}$ ): $87.94(\mathrm{~d}, J=$ $8.0 \mathrm{~Hz}, 2 \mathrm{H}), 7.78(\mathrm{~d}, J=8.0 \mathrm{~Hz}, 2 \mathrm{H}), 7.70(\mathrm{~d}, J=8.0 \mathrm{~Hz}, 2 \mathrm{H})$, $7.66(\mathrm{qd}, J=8.4 \mathrm{~Hz}, 4 \mathrm{H}), 7.20(\mathrm{~d}, J=8.8 \mathrm{~Hz}, 2 \mathrm{H}), 5.35(\mathrm{~s}$, $2 \mathrm{H}$ ), 4.61 (s, 2H); ${ }^{13} \mathrm{C}$ NMR (DMSO- $d_{6}$ ): $\delta 166.7,165.3,161.4$, 142.4, 141.8, 131.3, 130.1, 129.1, 128.7, 128.2, 128.0, 125.8, 125.8, 125.7, 123.3, 116.7, 115.2, 92.2, 69.0, 33.9. HRMS (ESI) (m/z): Calcd. for $\mathrm{C}_{27} \mathrm{H}_{17} \mathrm{~F}_{6} \mathrm{~N}_{3} \mathrm{O}_{2} \mathrm{~S}$, [M-H]+: 560.0867; found: 560.0870 .

\section{6-0xo-4-(4-phenoxyphenyl)-2-((4-(trifluoromethyl)} benzyl)thio)-1,6-dihydropyrimidine-5-carbonitrile (3m)

Yield 84\%; white solid; ${ }^{1} \mathrm{H}$ NMR (DMSO- $\left.d_{6}\right): \delta 7.93(\mathrm{~d}, J=$ $8.0 \mathrm{~Hz}, 2 \mathrm{H}), 7.66(\mathrm{qd}, J=8.0 \mathrm{~Hz}, 4 \mathrm{H}), 7.47(\mathrm{t}, J=7.2 \mathrm{~Hz}, 2 \mathrm{H})$, $7.24(\mathrm{t}, J=7.2 \mathrm{~Hz}, 1 \mathrm{H}), 7.14(\mathrm{~d}, J=7.6 \mathrm{~Hz}, 2 \mathrm{H}), 7.10(\mathrm{~d}, J=7.6$ $\mathrm{Hz}, 2 \mathrm{H}$ ), 4.58 (s, 2H); ${ }^{13} \mathrm{C}$ NMR (DMSO- $d_{6}$ ): $\delta 166.6,166.3$, 162.8, 160.4, 155.6, 142.7, 131.4, 130.7, 130.2, 130.1, 128.4, 128.1, 126.0, 125.8, 125.7, 125.1, 123.3, 120.3, 117.7, 117.0, 90.3, 33.8. HRMS (ESI) (m/z): Calcd. for $\mathrm{C}_{25} \mathrm{H}_{15} \mathrm{~F}_{3} \mathrm{~N}_{3} \mathrm{O}_{2} \mathrm{~S},[\mathrm{M}-\mathrm{H}]^{+}$: 478.0837; found: 478.0828 .

4-(2-((4-azidobenzyl)thio)-5-cyano-6-oxo-1,6-dihydropyrimidin-4-yl)-N-phenylbenzamide (3n)

Yield 65\%; white solid; ${ }^{1} \mathrm{H}$ NMR (DMSO- $\left.d_{6}\right): \delta 10.41(\mathrm{~s}, 1 \mathrm{H})$, $8.08(\mathrm{~d}, J=8.4 \mathrm{~Hz}, 2 \mathrm{H}), 8.01(\mathrm{~d}, J=8.0 \mathrm{~Hz}, 2 \mathrm{H}), 7.79(\mathrm{~d}, J=$ $8.0 \mathrm{~Hz}, 2 \mathrm{H}), 7.46(\mathrm{~d}, J=8.4 \mathrm{~Hz}, 2 \mathrm{H}), 7.37(\mathrm{t}, J=8.0 \mathrm{~Hz}, 2 \mathrm{H})$, $7.12(\mathrm{t}, J=7.6 \mathrm{~Hz}, 1 \mathrm{H}), 7.07(\mathrm{~d}, J=8.0 \mathrm{~Hz}, 2 \mathrm{H}), 4.48(\mathrm{~s}, 2 \mathrm{H})$; ${ }^{13}$ C NMR (DMSO- $d_{6}$ ): $\delta 167.8,167.1,165.5,163.0,139.4,138.8$, 137.8, 134.6, 131.1, 129.1, 128.2, 124.6, 120.8, 119.6, 117.0, 93.4, 43.0. HRMS (ESI) (m/z): Calcd. for $\mathrm{C}_{25} \mathrm{H}_{16} \mathrm{~N}_{7} \mathrm{O}_{2} \mathrm{~S},[\mathrm{M}-\mathrm{H}]^{+}$: 478.1086; found: 478.1099.

\section{Methyl4-(((5-cyano-6-oxo-4-(4-(phenylcarbamoyl)} phenyl)-1,6-dihydropyrimidin-2-yl)thio)methyl)benzoate (30)

Yield 54\%; white solid; ${ }^{1} \mathrm{H}$ NMR (DMSO- $d_{6}$ ): $\delta 10.41(\mathrm{~s}, 1 \mathrm{H}$ ), $8.08(\mathrm{~d}, J=8.4 \mathrm{~Hz}, 2 \mathrm{H}), 7.98(\mathrm{~d}, J=8.4 \mathrm{~Hz}, 2 \mathrm{H}), 7.91(\mathrm{~d}, J=$ $8.0 \mathrm{~Hz}, 2 \mathrm{H}), 7.79(\mathrm{~d}, J=8.0 \mathrm{~Hz}, 2 \mathrm{H}), 7.57(\mathrm{~d}, J=8.0 \mathrm{~Hz}, 2 \mathrm{H})$, $7.37(\mathrm{t}, J=8.0 \mathrm{~Hz}, 2 \mathrm{H}), 7.12(\mathrm{t}, J=7.6 \mathrm{~Hz}, 1 \mathrm{H}), 4.57(\mathrm{~s}, 2 \mathrm{H})$, 3.83 (s, $3 \mathrm{H}$ ); ${ }^{13} \mathrm{C}$ NMR (DMSO- $d_{6}$ ): $\delta 167.7,167.0,166.4,163.6$, 143.4, 139.4, 138.8, 137.7, 129.7, 129.1, 129.0, 128.9, 128.2, 124.4, 120.8, 117.0, 93.4, 52.5, 34.1. HRMS (ESI) (m/z): Calcd. for $\mathrm{C}_{27} \mathrm{H}_{19} \mathrm{~N}_{4} \mathrm{O}_{4} \mathrm{~S},[\mathrm{M}-\mathrm{H}]^{+}$: 495.1127 ; found: 495.1132.

4-(5-cyano-6-oxo-2-((4-(trifluoromethyl)benzyl)thio)1,6-dihydropyrimidin-4-yl)-N-phenylbenzamide (3p)

Yield 48\%; white solid; ${ }^{1} \mathrm{H}$ NMR (DMSO- $\left.d_{6}\right): \delta 10.41(\mathrm{~s}, 1 \mathrm{H})$, $8.08(\mathrm{~d}, J=8.0 \mathrm{~Hz}, 2 \mathrm{H}), 7.97(\mathrm{~d}, J=8.4 \mathrm{~Hz}, 2 \mathrm{H}), 7.79(\mathrm{~d}, J=$ $8.0 \mathrm{~Hz}, 2 \mathrm{H}), 7.67(\mathrm{qd}, J=8.0 \mathrm{~Hz}, 4 \mathrm{H}), 7.37(\mathrm{t}, J=8.0 \mathrm{~Hz}, 2 \mathrm{H})$, $7.12(\mathrm{t}, J=7.6 \mathrm{~Hz}, 1 \mathrm{H}), 4.56(\mathrm{~s}, 2 \mathrm{H}) ;{ }^{13} \mathrm{C}$ NMR (DMSO- $\left.d_{6}\right): \delta$ 167.7, 167.0, 165.3, 164.1, 143.0, 139.4, 138.9, 137.6, 130.1, 129.1, 129.0, 128.2, 125.7, 124.3, 123.3, 120.8, 117.1, 93.4, 33.9. HRMS (ESI) (m/z): Calcd. for $\mathrm{C}_{26} \mathrm{H}_{16} \mathrm{~F}_{3} \mathrm{~N}_{4} \mathrm{O}_{2} \mathrm{~S},[\mathrm{M}-\mathrm{H}]^{+}$: 505.0946; found: 505.0941 .

4-(2-((4-azidobenzyl)thio)-5-cyano-6-oxo-1,6-dihydropyrimidin-4-yl)-N-(4-chlorophenyl)benzamide (3q)

Yield 18\%; white solid; ${ }^{1} \mathrm{H}$ NMR (DMSO- $d_{6}$ ): $\delta 10.56$ (s, $1 \mathrm{H}$ ), $8.07(\mathrm{~d}, J=8.4 \mathrm{~Hz}, 2 \mathrm{H}), 7.98(\mathrm{~d}, J=8.0 \mathrm{~Hz}, 2 \mathrm{H}), 7.85(\mathrm{~d}, J=$ $8.8 \mathrm{~Hz}, 2 \mathrm{H}), 7.46(\mathrm{~d}, J=8.4 \mathrm{~Hz}, 2 \mathrm{H}), 7.43(\mathrm{~d}, J=8.8 \mathrm{~Hz}, 2 \mathrm{H})$, $7.07\left(\mathrm{~d}, J=8.4 \mathrm{~Hz}, 2 \mathrm{H}\right.$ ), 4.44 (s, 2H); ${ }^{13} \mathrm{C}$ NMR (DMSO- $d_{6}$ ): $\delta$ 168.5, 166.9, 165.4, 164.7, 139.3, 138.7, 138.5, 137.1, 134.9, 133.6, 131.0, 129.0, 128.2, 127.8, 127.1, 125.5, 123.9, 122.3, 119.7, 119.5, 117.5, 113.8, 111.7, 92.7, 33.9. HRMS (ESI) (m/z): Calcd. for $\mathrm{C}_{25} \mathrm{H}_{15} \mathrm{ClN}_{7} \mathrm{O}_{2} \mathrm{~S},[\mathrm{M}-\mathrm{H}]^{+}:$512.0696; found: 512.0698 .

Methyl4-(((4-(4-((4-chlorophenyl)carbamoyl)phenyl)5-cyano-6-oxo-1,6-dihydropyrimidin-2-yl)thio)methyl) benzoate ( $3 r$ )

Yield 31\%; white solid; ${ }^{1} \mathrm{H}$ NMR (DMSO- $d_{6}$ ): $\delta 10.55$ (s, $1 \mathrm{H}$ ), $8.08(\mathrm{~d}, J=8.4 \mathrm{~Hz}, 2 \mathrm{H}), 7.98(\mathrm{~d}, J=8.4 \mathrm{~Hz}, 2 \mathrm{H}), 7.90(\mathrm{~d}, J=$ $8.0 \mathrm{~Hz}, 2 \mathrm{H}), 7.84(\mathrm{~d}, J=8.8 \mathrm{~Hz}, 2 \mathrm{H}), 7.57(\mathrm{~d}, J=8.0 \mathrm{~Hz}, 2 \mathrm{H})$, $7.43(\mathrm{~d}, J=8.8 \mathrm{~Hz}, 2 \mathrm{H}), 4.57$ (s, 2H), 3.84 (s, 3H); ${ }^{13} \mathrm{C} \mathrm{NMR}$ (DMSO- $\left.d_{6}\right): \delta$ 167.6, 166.9, 166.4, 165.4, 163.7, 143.5, 139.0, 138.4, 137.3, 129.7, 129.3, 129.2, 129.1, 129.0, 128.9, 128.2, 127.9, 122.3, 117.0, 93.3, 52.58, 34.1. HRMS (ESI) (m/z): Calcd. for $\mathrm{C}_{27} \mathrm{H}_{18} \mathrm{ClN}_{4} \mathrm{O}_{4} \mathrm{~S},[\mathrm{M}-\mathrm{H}]^{+}$: 529.0737; found: 529.0734 .

$\mathrm{N}$-(4-chlorophenyl)-4-(5-cyano-6-0xo-2-((4(trifluoromethyl)benzyl)thio)-1,6-dihydropyrimidin-4-yl) benzamide (3s)

Yield 17\%; white solid; ${ }^{1} \mathrm{H}$ NMR (DMSO- $d_{6}$ ): $\delta 10.55$ (s, $1 \mathrm{H}), 8.05(\mathrm{~d}, J=8.0 \mathrm{~Hz}, 2 \mathrm{H}), 7.94(\mathrm{~d}, J=8.4 \mathrm{~Hz}, 2 \mathrm{H}), 7.84$ 
(d, $J=8.8 \mathrm{~Hz}, 2 \mathrm{H}), 7.67(\mathrm{qd}, J=8.0 \mathrm{~Hz}, 4 \mathrm{H}), 7.57(\mathrm{~d}, J=$ $8.0 \mathrm{~Hz}, 2 \mathrm{H}), 7.43$ (d, $J=8.8 \mathrm{~Hz}, 2 \mathrm{H}), 4.52(\mathrm{~s}, 2 \mathrm{H}) ;{ }^{13} \mathrm{C} \mathrm{NMR}$ $\left(\mathrm{DMSO}-d_{6}\right): \delta 168.2,166.9,165.4,143.1,139.1,138.4,137.30$, 130.1, 129.0,128.2, 127.9, 127.1, 126.0, 125.8, 125.7, 122.3, 117.2, 93.1, 33.8. HRMS (ESI) (m/z): Calcd. for $\mathrm{C}_{26} \mathrm{H}_{15} \mathrm{ClF}_{3} \mathrm{~N}_{4} \mathrm{O}_{2} \mathrm{~S}$, $[\mathrm{M}-\mathrm{H}]^{+}$: 539.0556; found: 539.0541.

\section{4-(2-((4-azidobenzyl)thio)-5-cyano-6-oxo-1,6-dihydropy- rimidin-4-yl)-N-(p-tolyl)benzamide (3t)}

Yield 51\%; white solid; ${ }^{1} \mathrm{H}$ NMR (DMSO- $\left.d_{6}\right): \delta 10.34(\mathrm{~s}, 1 \mathrm{H})$, 8.08 (d, $J=8.4 \mathrm{~Hz}, 2 \mathrm{H}), 8.03$ (d, $J=8.4 \mathrm{~Hz}, 2 \mathrm{H}), 7.67$ (d, $J$ $=8.0 \mathrm{~Hz}, 2 \mathrm{H}), 7.46(\mathrm{~d}, J=8.0 \mathrm{~Hz}, 2 \mathrm{H}), 7.17(\mathrm{~d}, J=8.4 \mathrm{~Hz}$, 2H), 7.07 (d, $J=8.0 \mathrm{~Hz}, 2 \mathrm{H}), 4.51$ (s, 2H); ${ }^{13} \mathrm{C}$ NMR (DMSO$\left.d_{6}\right): \delta 167.1,165.2,138.9,137.8,136.7,134.6,133.5,129.5,129.0$, 128.2, 120.8, 120.0, 119.6, 116.7, 93.4, 34.0, 20.9. HRMS (ESI) (m/z): Calcd. for $\mathrm{C}_{26} \mathrm{H}_{18} \mathrm{~N}_{7} \mathrm{O}_{2} \mathrm{~S},[\mathrm{M}-\mathrm{H}]^{+}$: 492.1243; found: 492.1250.

\section{Methyl-4-(((5-cyano-6-0xo-4-(4-(p-tolylcarbamoyl) phenyl)-1,6-dihydropyrimidin-2-yl)thio)methyl)benzoate} (3u)

Yield 62\%; white solid; ${ }^{1} \mathrm{H}$ NMR (DMSO- $d_{6}$ ): $\delta 10.34$ (s, 1H), 8.07 (d, $J=8.0 \mathrm{~Hz}, 2 \mathrm{H}), 7.98$ (d, $J=8.0 \mathrm{~Hz}, 2 \mathrm{H}), 7.91$ $(\mathrm{d}, J=8.0 \mathrm{~Hz}, 2 \mathrm{H}), 7.67(\mathrm{~d}, J=8.0 \mathrm{~Hz}, 2 \mathrm{H}), 7.58(\mathrm{~d}, J=$ $8.4 \mathrm{~Hz}, 2 \mathrm{H}), 7.17$ (d, $J=8.0 \mathrm{~Hz}, 2 \mathrm{H}), 4.58$ (s, 2H), 3.83 (s, $3 \mathrm{H}) ;{ }^{13} \mathrm{C}$ NMR (DMSO- $\left.d_{6}\right): \delta 167.5,167.0,166.4,165.1,143.5$, 138.7, 137.7, 136.9, 133.3, 132.1, 129.9, 129.7, 129.5, 129.0, 128.9, 128.1, 117.0, 93.3, 52.5, 34.1, 20.9. HRMS (ESI) (m/z): Calcd. for $\mathrm{C}_{28} \mathrm{H}_{21} \mathrm{~N}_{4} \mathrm{O}_{4} \mathrm{~S},[\mathrm{M}-\mathrm{H}]^{+}$: 509.1278; found: 509.1277 .

\section{4-(5-cyano-6-oxo-2-((4-(trifluoromethyl)benzyl)thio)- 1,6-dihydropyrimidin-4-yl)- $\mathrm{N}$-(p-tolyl)benzamide (3v)}

Yield 45\%; white solid; ${ }^{1} \mathrm{H}$ NMR (DMSO- $\left.d_{6}\right): \delta 10.34(\mathrm{~s}, 1 \mathrm{H})$, $8.07(\mathrm{~d}, J=8.4 \mathrm{~Hz}, 2 \mathrm{H}), 7.97$ (d, $J=8.4 \mathrm{~Hz}, 2 \mathrm{H}), 7.64-7.71(\mathrm{~m}$, $6 \mathrm{H}), 7.16(\mathrm{~d}, J=8.4 \mathrm{~Hz}, 2 \mathrm{H}), 4.59$ (s, 2H); ${ }^{13} \mathrm{C}$ NMR (DMSO$\left.d_{6}\right): \delta 167.1,167.0,165.1,162.8,142.7,138.5,137.8,136.9,133.3$, 130.2, 129.4, 129,0, 128.5, 128.2, 125.7, 123.5, 120.8, 116.7, 93.7, 33.9, 20.9. HRMS (ESI) (m/z): Calcd. for $\mathrm{C}_{27} \mathrm{H}_{18} \mathrm{~F}_{3} \mathrm{~N}_{4} \mathrm{O}_{2} \mathrm{~S}$, $[\mathrm{M}-\mathrm{H}]^{+}$: 519.1108; found: 519.1094 .

Acknowledgments: FB was a visiting scholar at GSU when conducting the lab research with partial financial support from the Islamic Development Bank (IDB) under a merit scholarship programme. Part of the research was conducted with the partial financial support of the National Institute of Health awarded to PCT and BW (AI104168).

\section{References}

[1] Ventola CL. The antibiotic resistance crisis: part 1: causes and threats. P\&T. 2015; 40: 277-83.

[2] Magiorakos AP, Srinivasan A, Carey RB, Carmeli Y, Falagas ME, Giske CG, et al. Multidrug-resistant, extensively drug-resistant and pandrug-resistant bacteria: an international expert proposal for interim standard definitions for acquired resistance. Clin Microbiol Infect. 2012; 18: 268-81.

[3] Willyard C. The drug-resistant bacteria that pose the greatest health threats. Nature. 2017; 543:15.

[4] Antibiotic / Antimicrobial Resistance (AR / AMR) https:// http://www.cdc.gov/drugresistance/index.html

[5] Zimmer J, Nam Y, Rapoport TA. Structure of a complex of the ATPase SecA and the protein-translocation channel. Nature. 2008; 455: 936-43.

[6] Driessen AJ, Fekkes P, van der Wolk JP. The Sec system. Curr Opin Microbiol. 1998; 1: 216-22.

[7] Hsieh YH, Zhang H, Lin BR, Cui N, Na B, Yang H, et al. SecA alone can promote protein translocation and ion channel activity: SecYEG increases efficiency and signal peptide specificity. J Biol Chem. 2011; 286: 44702-9.

[8] Wang H, Ma Y, Hsieh YH, Yang H, Li M, Wang B, et al. Trimer Is Functional in the Membranes: Existence of Higher Oligomers? Biochem. Biophys. Res Commun. 2014; 447: 250-4.

[9] Chaudhary AS, Chen W, Jin J, Tai PC, Wang B. SecA: a potential antimicrobial target. Future Med Chem. 2015; 7: 989-1007.

[10] Sugie Y, Inagaki S, Kato Y, Nishida H, Pang CH, Saito T, et al. CJ-21,058, a new SecA inhibitor isolated from a fungus. J Antibiot (Tokyo). 2002; 55: 25-9.

[11] Parish CA, de la Cruz M, Smith SK, Zink D, Baxter J, TuckerSamaras S, et al. Antisense-guided isolation and structure elucidation of pannomycin, a substituted cis-decalin from Geomyces pannorum. J Nat Prod. 2009; 72: 59-62.

[12] Jang MY, De Jonghe S, Segers K, Anne J, Herdewijn P. Synthesis of novel 5-amino-thiazolo[4,5-d]pyrimidines as E. coli and S. aureus SecA inhibitors. Bioorg Med Chem. 2011; 19: 702-14.

[13] Akula N, Trivedi P, Han FQ, Wang N. Identification of small molecule inhibitors against SecA of Candidatus Liberibacter asiaticus by structure based design. Eur J Med Chem. 2012; 54: 919-24.

[14] Akula N, Zheng H, Han FQ, Wang N. Discovery of novel SecA inhibitors of Candidatus Liberibacter asiaticus by structure based design. Bioorg Med Chem Lett. 2011; 21: 4183-8.

[15] Li M, Huang Y], Tai PC, Wang B. Discovery of the first SecA inhibitors using structure-based virtual screening. Biochem Biophys Res Commun. 2008; 368: 839-45.

[16] Chen W, Huang YJ, Gundala SR, Yang H, Li M, Tai PC, et al. The first low $\mu M$ SecA inhibitors. Bioorg Med Chem. 2010; 18: 1617-25.

[17] Huang YJ, Wang H, Gao FB, Li M, Yang H, Wang B, et al. Fluorescein analogues inhibit SecA ATPase: the first 
sub-micromolar inhibitor of bacterial protein translocation. ChemMedChem. 2012; 7: 571-7.

[18] Cui J, Jin J, Hsieh YH, Yang H, Ke B, Damera K, et al. Design, synthesis and biological evaluation of rose bengal analogues as SecA inhibitors. ChemMedChem. 2013; 8: 1384-93.

[19] Chaudhary AS, Jin J, Chen W, Tai PC, Wang B. Design, syntheses and evaluation of 4-0xo-5-cyano thiouracils as SecA inhibitors. Bioorg Med Chem. 2015; 23: 105-17.

[20] Ullah S, Kang D, Lee S, Ikram M, Park C, Park Y, et al. Synthesis of cinnamic amide derivatives and their anti-melanogenic effect in alpha-MSH-stimulated B16F10 melanoma cells. Eur J Med Chem. 2019; 161: 78-92.

[21] Arienti KL, Brunmark A, Axe FU, McClure K, Lee A, Blevitt J, et al. Checkpoint kinase inhibitors: SAR and radioprotective properties of a series of 2-arylbenzimidazoles. J Med Chem. 2005; 48: 1873-85.

[22] Cui J, Jin J, Chaudhary AS, Hsieh YH, Zhang H, Dai C, et al. Design, Synthesis and Evaluation of Triazole-Pyrimidine Analogues as SecA Inhibitors. ChemMedChem. 2016; 11: 43-56. 